\title{
Recalibration of the $M_{\mathrm{BH}}-\sigma_{\star}$ Relation for AGN
}

\author{
Merida Batiste ${ }^{1}$, Misty C. Bentz ${ }^{1}$, Sandra I. Raimundo ${ }^{2}$, Marianne Vestergaard ${ }^{2,3}$, and Christopher A. Onken ${ }^{4}$ \\ ${ }^{1}$ Department of Physics \& Astronomy, Georgia State University, 25 Park Place, Atlanta, GA 30303, USA; batiste@ astro.gsu.edu \\ 2 Dark Cosmology Centre, Niels Bohr Institute, University of Copenhagen, Juliane Maries Vej 30, DK-2100 Copenhagen Ø, Denmark \\ ${ }^{3}$ Department of Astronomy and Steward Observatory, University of Arizona, 933 N. Cherry Avenue, Tucson, AZ 85721, USA \\ ${ }^{4}$ Research School of Astronomy \& Astrophysics, The Australian National University, Canberra, ACT 2611, Australia \\ Received 2016 November 18; revised 2017 March 6; accepted 2017 March 8; published 2017 March 24
}

\begin{abstract}
We present a recalibration of the $M_{\mathrm{BH}}-\sigma_{\star}$ relation, based on a sample of 16 reverberation-mapped galaxies with newly determined bulge stellar velocity dispersions $\left(\sigma_{\star}\right)$ from integral-field spectroscopy (IFS), and a sample of 32 quiescent galaxies with publicly available IFS. For both samples, $\sigma_{\star}$ is determined via two different methods that are popular in the literature, and we provide fits for each sample based on both sets of $\sigma_{\star}$. We find the fit to the active galactic nucleus sample is shallower than the fit to the quiescent galaxy sample, and that the slopes for each sample are in agreement with previous investigations. However, the intercepts to the quiescent galaxy relations are notably higher than those found in previous studies, due to the systematically lower $\sigma_{\star}$ measurements that we obtain from IFS. We find that this may be driven, in part, by poorly constrained measurements of bulge effective radius $\left(r_{e}\right)$ for the quiescent galaxy sample, which may bias the $\sigma_{\star}$ measurements low. We use these quiescent galaxy parameterizations, as well as one from the literature, to recalculate the virial scaling factor $f$. We assess the potential biases in each measurement, and suggest $f=4.82 \pm 1.67$ as the best currently available estimate. However, we caution that the details of how $\sigma_{\star}$ is measured can significantly affect $f$, and there is still much room for improvement.
\end{abstract}

Key words: galaxies: active - galaxies: bulges - galaxies: kinematics and dynamics

\section{Introduction}

A wealth of evidence demonstrates that the formation and evolution of galaxies and their supermassive black holes (BHs) are fundamentally connected. This connection is exemplified by empirically determined scaling relations between the mass of a central $\mathrm{BH}, M_{\mathrm{BH}}$, and host galaxy properties, including bulge stellar velocity dispersion, $\sigma_{\star}$ (Ferrarese \& Merritt 2000; Gebhardt et al. 2000). These scaling relations provide insight into the mechanisms governing the formation and evolution of structure and may be used to estimate $M_{\mathrm{BH}}$ for large samples of galaxies at cosmological distances.

Accurate calibration of scaling relations requires a sample of galaxies with secure $M_{\mathrm{BH}}$ determinations. In quiescent galaxies, this is usually done by modeling the spatially resolved gas or stellar kinematics within the gravitational sphere of influence of the $\mathrm{BH}$ and is thus limited to the local universe. To probe $M_{\mathrm{BH}}$ over cosmological distances requires active galactic nuclei (AGN), for which $M_{\mathrm{BH}}$ can be determined via reverberationmapping (RM; Blandford \& McKee 1982). RM exploits the variability of the AGN to probe the gas in the broad-line region (BLR) around the BH. A dimensionless scale factor $f$ is necessary for this method, to account for the unknown geometry and kinematics of the BLR. Since direct determination of $f$ is rarely feasible, it is assumed that AGN and quiescent galaxies follow the same $M_{\mathrm{BH}}-\sigma_{\star}$ relation. The value of $f$ is then estimated as the average multiplicative offset required to bring the relations for $\mathrm{AGN}$ and quiescent galaxies into agreement (Onken et al. 2004). Accurate calibration of the $M_{\mathrm{BH}}-\sigma_{\star}$ relation is, therefore, essential for $\mathrm{RM} M_{\mathrm{BH}}$ determinations.

The $M_{\mathrm{BH}}-\sigma_{\star}$ relation appears to be the tightest and most fundamental of the observed scaling relations (e.g., Beifiori et al. 2012; Shankar et al. 2016), and has consequently been the subject of extensive investigation (see reviews by Kormendy \&
Ho 2013, hereafter KH13, and Graham 2016). However, it remains unclear what the actual best-fitting relation is, or indeed whether a single relation holds, for both active and quiescent galaxies. Studies suggest a significant difference between the slopes of the relation for quiescent galaxies (McConnell \& Ma 2013) and AGN (Woo et al. 2010); however, simulations indicate that this may simply be an artifact of sample selection bias (Woo et al. 2013; hereafter W13; Shankar et al. 2016).

Studies further indicate a possible morphological dependence of the $M_{\mathrm{BH}}-\sigma_{\star}$ relation. In particular, that galaxies with substructure such as bars and pseudo-bulges are offset from the elliptical-only relation (e.g., Graham 2008; Hu 2008; Gültekin et al. 2009). This is particularly relevant when measuring $\sigma_{\star}$ for AGN, which is often done via single-aperture and long-slit spectroscopy. Contamination by dynamically distinct substructure is usually unavoidable, and rotational broadening due to disk contamination can strongly affect $\sigma_{\star}$ measurements from single-aperture spectra (e.g., Graham et al. 2011; Bellovary et al. 2014; Hartmann et al. 2014; W13). In addition, Batiste et al. (2017, hereafter B17) showed that slit orientation relative to substructure, such as bars, can strongly affect the measured $\sigma_{\star}$. These issues preferentially impact the spiral-dominated local RM AGN sample, thereby inhibiting investigation of possible differences between quiescent and active galaxies.

This problem is mitigated by spatially resolved kinematics from integral-field spectroscopy (IFS), which allows for significant improvement in $\sigma_{\star}$ determinations. B17 provide IFS-based $\sigma_{\star}$ estimates for $10 \mathrm{RM}$ AGN, and IFS is available in the literature for a further 6. IFS is also available for 32 quiescent galaxies with dynamical $M_{\mathrm{BH}}$ measurements. In this Letter, we use these samples to re-calibrate the $M_{\mathrm{BH}}-\sigma_{\star}$ relation for quiescent galaxies and $\mathrm{AGN}$ and provide a new estimate of the scale factor $f$ for use with $\mathrm{RM} M_{\mathrm{BH}}$ determination. 
Table 1

AGN Sample

\begin{tabular}{|c|c|c|c|c|c|c|c|c|}
\hline $\begin{array}{l}\text { Object } \\
\text { (1) }\end{array}$ & $\begin{array}{l}r_{e} \\
\left({ }^{\prime \prime}\right) \\
(2)\end{array}$ & $\begin{array}{c}\text { References } \\
\text { (3) }\end{array}$ & $\begin{array}{c}\sigma_{\star} \\
\left(\mathrm{km} \mathrm{s}^{-1}\right) \\
(4)\end{array}$ & $\begin{array}{c}\sigma_{\star_{\text {int }}} \\
\left(\mathrm{km} \mathrm{s}^{-1}\right) \\
(5)\end{array}$ & $\begin{array}{l}\text { Std Deviation } \\
\left(\mathrm{km} \mathrm{s}^{-1}\right) \\
(6)\end{array}$ & $\begin{array}{c}\text { References } \\
\text { (7) }\end{array}$ & $\begin{array}{c}\mathrm{VP} \\
\left(10^{7} M_{\odot}\right) \\
(8)\end{array}$ & $\begin{array}{l}\text { Morphological Type } \\
\text { (9) }\end{array}$ \\
\hline Mrk 79 & 2.0 & 1 & $120 \pm 9$ & $125 \pm 15$ & 21 & 6 & $0.951_{-0.256}^{+0.267}$ & barred late \\
\hline NGC 3516 & 2.1 & 5 & $139 \pm 4$ & $143 \pm 4$ & 12 & 6 & $0.577_{-0.076}^{+0.051}$ & barred late \\
\hline NGC 4051 & 1.0 & 5 & $74 \pm 2$ & $69 \pm 4$ & 4 & 6 & $0.031_{-0.009}^{+0.010}$ & barred late \\
\hline NGC 4593 & 11.5 & 5 & $113 \pm 3$ & $144 \pm 5$ & 14 & 6 & $0.177_{-0.038}^{+0.038}$ & barred late \\
\hline NGC 5273 & 6.8 & 3 & $62 \pm 3$ & $69 \pm 5$ & 9 & 8 & $0.103_{-0.076}^{+0.057}$ & early \\
\hline Mrk 279 & 1.6 & 1 & $153 \pm 7$ & $156 \pm 17$ & 26 & 6 & $0.657_{-0.177}^{+0.177}$ & early \\
\hline PG $1411+442$ & 3.1 & 1 & $\ldots$ & $208 \pm 30$ & $\ldots$ & 7 & $6.263_{-3.376}^{+3.344}$ & early \\
\hline NGC 5548 & 11.2 & 5 & $131 \pm 3$ & $162 \pm 12$ & 34 & 6 & $1.212_{-0.050}^{+0.052}$ & late \\
\hline PG $1617+175$ & 1.7 & 1 & $\ldots$ & $201 \pm 37$ & $\ldots$ & 7 & $9.620_{-4.790}^{+4.272}$ & early \\
\hline
\end{tabular}

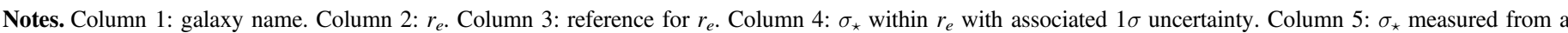

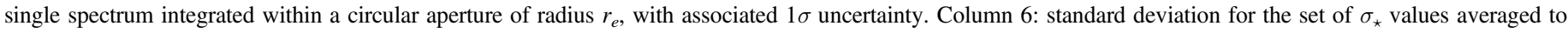

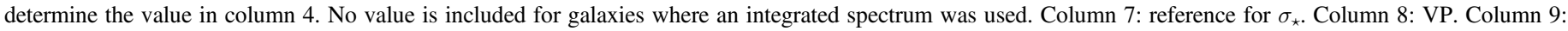
morphological type, based on surface brightness decompositions of Bentz et al. (2009, 2013) and M. C. Bentz et al. (2017, in preparation).

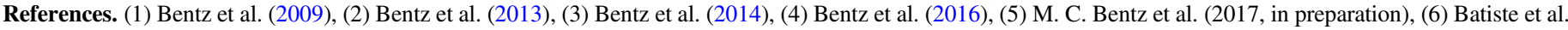
(2017), (7) Grier et al. (2013), (8) this work.

Throughout this work, we adopt a $\Lambda$ CDM cosmology with $\Omega_{m}=0.3, \Omega_{\Lambda}=0.7$, and $H_{0}=70 \mathrm{~km} \mathrm{~s}^{-1} \mathrm{Mpc}^{-1}$.

\section{The AGN Sample}

We include in our analysis all 16 RM AGN host galaxies that have so far been observed with IFS. While this is a small subset of the full RM AGN sample, the rest of which are targets of an ongoing observational campaign, it does provide a representative overview of the full sample for the $\sigma_{\star}$ and $M_{\mathrm{BH}}$ ranges probed.

\subsection{Virial Products (VPs) from Reverberation-mapping}

RM allows accurate determination of the VP, given by $\mathrm{VP}=V^{2} R_{\mathrm{BLR}} / G$, where $G$ is the gravitational constant, $V$ is measured from the width of a broad emission line, and $R_{\mathrm{BLR}}$ is the size of the BLR. VPs are drawn from the AGN BH Mass Database $^{5}$ (Bentz \& Katz 2015) and from Bentz et al. (2016), and are listed in column (7) of Table 1. In all cases, VP is determined from the $\mathrm{H} \beta$ line. Individual references are available from the database.

\subsection{Bulge Stellar Velocity Dispersions}

Spatially resolved stellar kinematics are available for NGC 5273 , from the ATLAS ${ }^{3 \mathrm{D}}$ survey of early-type galaxies in the northern hemisphere ${ }^{6}$ (Cappellari et al. 2011, 2013), and for MGC-06-30-15, from the work of Raimundo et al. (2013). Following the method of B17, $\sigma_{\star}$ is determined for these galaxies by taking an error-weighted average of the values for

\footnotetext{
http://www.astro.gsu.edu/AGNmass/

6 http://www-astro.physics.ox.ac.uk/atlas3d/
}

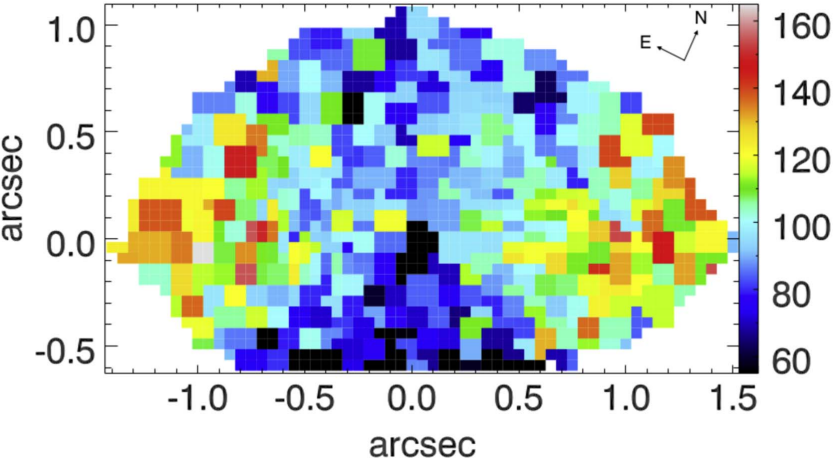

Figure 1. Map of $\sigma_{\star}$ for MCG-06-30-15, based on data from Raimundo et al. (2013).

each spaxel within a circular aperture defined by the effective radius, $r_{e}$.

Accurate measurements of $r_{e}$ are available from the works of Bentz et al. (2014) for NGC 5273 and Bentz et al. (2016) for MGC-06-30-15. They are determined from detailed surface brightness decompositions of Hubble Space Telescope (HST) images, using GALFIT (Peng et al. 2002, 2010). The AGN is isolated from the galaxy surface brightness features, and substructure such as bars and disks are accounted for.

For MCG-06-30-15, we follow Raimundo et al. (2013) and exclude the central 0 "' 1 from our calculation of $\sigma_{\star}$, as the noise associated with the AGN continuum precludes secure measurement of the stellar kinematics. Furthermore, the kinematic map in Figure 1 shows that a small region within $r_{e}(1 . \prime 01)$ was cut off ( $y \leqslant-0$ " 6 , the bottom of the map), due to an illumination artifact in the SINFONI data (see Raimundo et al. 2013 for details). We assume that the kinematics within $r_{e}$ are well 
represented by the region that remains and determine $\sigma_{\star}$ without applying any correction.

Two estimates of uncertainty are provided for each $\sigma_{\star}$ determination. The statistical uncertainty, based on the measurement error, is shown in column (4) of Table 1 along with $\sigma_{\star}$. Column (6) shows the standard deviation among the set of $\sigma_{\star}$ values that have been averaged to determine the overall $\sigma_{\star}$. The standard deviation provides a measure of the spatial variation in the kinematics within $r_{e}$ and may be more physically meaningful as an estimate of uncertainty.

Finally, $\sigma_{\star}$ determinations from IFS are available for four high-luminosity quasar hosts from Grier et al. (2013). This study employs a different definition of $\sigma_{\star}$, which includes a contribution from the rotational velocity. Rather than averaging the kinematics within a chosen aperture, the spectra within that aperture are instead co-added, and $\sigma_{\star}$ is measured from the resulting rotationally broadened spectrum. While this method differs from that of B17, it has been favored in some recent studies, including those using IFS (e.g., Gültekin et al. 2009; Cappellari et al. 2013; van den Bosch 2016; KH13). For elliptical or near face-on disk galaxies the difference between the methods should be minimal, since rotation along the line of sight will not dominate the stellar kinematics (W13). Based on the GALFIT decompositions of Bentz et al. (2009), two of these quasar hosts are elliptical (i.e., fitted with only a bulge component) and two are low-inclination disk galaxies. Consequently, we do not expect any bias to arise from including these measurements.

We can directly test this expectation because our sample contains galaxies qualitatively similar to the quasar hosts, including the low-inclination disk galaxies NGC 5273 and NGC 6814. We measure $\sigma_{\star}$ for the whole sample via the method of Grier et al. (2013) (column (5) of Table 1). The greatest variation between $\sigma_{\star}$ estimates from the two methods occurs for inclined spiral galaxies with significant substructure (e.g., NGC 4593) or evidence of ongoing or recent interactions (e.g., NGC 3227, MCG-06-30-15, and NGC 5548; M. C. Bentz et al. 2017, in preparation). As expected, $\sigma_{\star}$ varies minimally between the two methods for NGC 5273 and NGC 6814, suggesting that including the quasars is unlikely to introduce significant bias.

Measurements of $\sigma_{\star}$ for the full sample are given in Table 1 .

\section{The Quiescent Galaxy Sample}

Cappellari et al. (2013) provide stellar kinematics for 32 quiescent galaxies from the compilation of KH13 (listed in Table 2), with $\sigma_{\star}$ determined via the same method as Grier et al. (2013). For comparison, we calculate $\sigma_{\star}$ for each galaxy following the method of B17, using $r_{e}$ measurements from Cappellari et al. (2011). The sample contains elliptical and disk galaxies, so these methods give quite different results in some cases. On average, the measurements of Cappellari et al. (2013) are larger, by $\sim 13 \mathrm{~km} \mathrm{~s}^{-1}$.

It is essential to note here that while the stellar kinematics are high quality, the measurements of $r_{e}$ are less reliable (see discussion by Cappellari et al. 2013). Measurements come from seeing-limited ground-based images, rather than HST images, as are used for the AGN sample. Moreover, $r_{e}$ was defined as the radius containing half the observed light for the whole galaxy (Cappellari et al. 2013), rather than for the bulge. For the S0 galaxy NGC 5273, we find that the value quoted by Cappellari et al. (2011) $\left(r_{e}=37\right.$ !' 15) is more than $5 \times$ larger
Table 2

Quiescent Galaxies

\begin{tabular}{|c|c|c|c|c|}
\hline $\begin{array}{l}\text { Galaxy } \\
\text { (1) }\end{array}$ & $\begin{array}{c}\sigma_{\star \mathrm{ATLAS}} \\
\left(\mathrm{km} \mathrm{s}^{-1}\right) \\
(2)\end{array}$ & $\begin{array}{c}\sigma_{\star_{\text {call }}} \\
\left(\mathrm{km} \mathrm{s}^{-1}\right) \\
(3)\end{array}$ & $\begin{array}{c}\text { Standard } \\
\text { Deviation } \\
\left(\mathrm{km} \mathrm{s}^{-1}\right) \\
(4)\end{array}$ & $\begin{array}{l}M_{\mathrm{BH}} \\
\left(10^{7} M_{\odot}\right) \\
(5)\end{array}$ \\
\hline NGC 0524 & $220 \pm 11$ & $206 \pm 12$ & 22 & $86.7_{-4.6}^{+9.4}$ \\
\hline NGC 0821 & $179 \pm 9$ & $173 \pm 10$ & 17 & $16.5_{-7.3}^{+7.4}$ \\
\hline NGC 1023 & $167 \pm 8$ & $145 \pm 7$ & 34 & $4.1_{-0.4}^{+0.4}$ \\
\hline NGC 2549 & $142 \pm 7$ & $109 \pm 10$ & 19 & $1.5_{-1.1}^{+0.2}$ \\
\hline NGC 2778 & $132 \pm 7$ & $112 \pm 11$ & 28 & $1.5_{-1.5}^{+1.5}$ \\
\hline NGC 3245 & $177 \pm 9$ & $126 \pm 9$ & 30 & $23.9_{-7.6}^{+2.7}$ \\
\hline NGC 3377 & $128 \pm 6$ & $105 \pm 10$ & 19 & $17.8_{-9.3}^{+9.4}$ \\
\hline NGC 3379 & $186 \pm 9$ & $185 \pm 9$ & 16 & $41.6_{-10.4}^{+10.4}$ \\
\hline NGC 3384 & $138 \pm 7$ & $118 \pm 7$ & 19 & $1.1_{-0.5}^{+0.5}$ \\
\hline NGC 3489 & $101 \pm 5$ & $74 \pm 8$ & 14 & $0.6_{-0.1}^{+0.1}$ \\
\hline NGC 3607 & $207 \pm 10$ & $206 \pm 11$ & 17 & $13.7_{-4.7}^{+4.5}$ \\
\hline NGC 3608 & $169 \pm 8$ & $166 \pm 10$ & 17 & $46.5_{-9.9}^{+9.9}$ \\
\hline NGC 3945 & $177 \pm 9$ & $141 \pm 10$ & 25 & $0.9_{-0.9}^{+1.7}$ \\
\hline NGC 3998 & $224 \pm 11$ & $182 \pm 9$ & 34 & $84.5_{-6.6}^{+7.0}$ \\
\hline NGC 4026 & $157 \pm 8$ & $123 \pm 9$ & 23 & $18.0_{-3.5}^{+6.0}$ \\
\hline NGC 4261 & $265 \pm 13$ & $285 \pm 11$ & 22 & $52.9_{-10.8}^{+10.7}$ \\
\hline NGC 4342 & $242 \pm 12$ & $192 \pm 7$ & 29 & $45.3_{-14.8}^{+26.5}$ \\
\hline NGC 4374 & $258 \pm 13$ & $271 \pm 9$ & 20 & $92.5_{-8.7}^{+9.8}$ \\
\hline NGC 4382 & $179 \pm 9$ & $190 \pm 7$ & 11 & $1.3_{-1.3}^{+21.1}$ \\
\hline NGC 4459 & $158 \pm 8$ & $135 \pm 10$ & 20 & $7.0_{-1.3}^{+1.3}$ \\
\hline NGC 4472 & $250 \pm 13$ & $266 \pm 7$ & 18 & $254.0_{-10.0}^{+58.0}$ \\
\hline NGC 4473 & $187 \pm 9$ & $176 \pm 8$ & 22 & $9.0_{-4.5}^{+4.5}$ \\
\hline $\begin{array}{l}\text { NGC } \\
\quad 4486 \text { (M87) }\end{array}$ & $264 \pm 13$ & $295 \pm 4$ & 19 & $615.0_{-37.0}^{+38.0}$ \\
\hline NGC 4486A & $123 \pm 6$ & $115 \pm 16$ & 71 & $1.4_{-0.5}^{+0.5}$ \\
\hline NGC 4526 & $209 \pm 10$ & $175 \pm 7$ & 30 & $45.1_{-10.3}^{+14.0}$ \\
\hline NGC 4564 & $155 \pm 8$ & $134 \pm 9$ & 24 & $8.8_{-2.4}^{+2.5}$ \\
\hline NGC 4596 & $126 \pm 6$ & $127 \pm 10$ & 31 & $7.7_{-3.2}^{+3.7}$ \\
\hline NGC 4649 & $268 \pm 13$ & $283 \pm 7$ & 22 & $472.0_{-105.0}^{+104.0}$ \\
\hline NGC 4697 & $169 \pm 8$ & $166 \pm 9$ & 13 & $20.2_{-5.0}^{+5.1}$ \\
\hline NGC 5576 & $155 \pm 8$ & $155 \pm 10$ & 22 & $27.3_{-7.9}^{+6.8}$ \\
\hline NGC 5845 & $228 \pm 11$ & $178 \pm 6$ & 35 & $48.7_{-15.3}^{15.3}$ \\
\hline NGC 7457 & $75 \pm 4$ & $62 \pm 11$ & 15 & $0.9_{-0.5}^{+0.5}$ \\
\hline
\end{tabular}

Notes. Column 1: galaxy name. Column 2: $\sigma_{\star}$ from Cappellari et al. (2013), measured from a single spectrum integrated within $r_{e}$. Column 3: $\sigma_{\star}$ within $r_{e}$ determined from kinematic maps of Cappellari et al. (2011) with associated $1 \sigma$ uncertainty. Column 4: standard deviation for the set of $\sigma_{\star}$ values averaged to determine the value in column 3. Column 5: $M_{\mathrm{BH}}$ from the compilation of KH13.

than that determined from the bulge-disk decompositions of Bentz et al. (2014).

Falcón-Barroso et al. (2017) have shown that, for early-type galaxies (including lenticulars and $\mathrm{Sa}$ galaxies), $\sigma_{\star}$ generally decreases with radius. Consequently, if we assume that $r_{e}$ is typically overestimated by $\sim 5 \times$ for the quiescent sample, then the corresponding $\sigma_{\star}$ measurements are likely biased low. However, for inclined disk galaxies the inclusion of disk rotation at large radii may bias the estimates high (e.g., Bellovary et al. 2014). The quiescent sample contains a range of galaxy morphologies and disk inclinations, so there are multiple reasons to be cautious with the adopted $r_{e}$ values and the quoted $\sigma_{\star}$ measurements.

Since similar measurements of $r_{e}$ have been used in previous studies (e.g., Gültekin et al. 2009; KH13), it is safe to assume 
that all studies of the $M_{\mathrm{BH}}-\sigma_{\star}$ relation are affected by this issue to some extent, and substantially improved measurements of $r_{e}$ will be critical to all future efforts to properly calibrate the $M_{\mathrm{BH}}-\sigma_{\star}$ relation.

\section{The $M_{\mathrm{BH}^{-}}-\sigma_{\star}$ Relation}

The $M_{\mathrm{BH}}-\sigma_{\star}$ relation is parameterized as

$$
\log \left(\frac{M_{\mathrm{BH}}}{M_{\odot}}\right)=\alpha+\beta \log \left(\frac{\sigma_{\star}}{200 \mathrm{~km} \mathrm{~s}^{-1}}\right) .
$$

We fit a standard forward regression using the LINMIX_ERR routine of Kelly (2007), which employs a fully Bayesian approach. We also tested the popular MPFITEXY routine of Williams et al. (2010), since Park et al. (2012) showed that both are similarly robust and unbiased, and found that the results are consistent with those determined by LINMIX_ERR.

\subsection{The Quiescent Galaxy Sample}

Using $\sigma_{\star}$ from Cappellari et al. (2013), we find a best-fitting relation for the quiescent sample of

$$
\begin{aligned}
\log \left(\frac{M_{\mathrm{BH}}}{M_{\odot}}\right)= & (8.55 \pm 0.09) \\
& +(5.32 \pm 0.63) \log \left(\frac{\sigma_{\star}}{200 \mathrm{~km} \mathrm{~s}^{-1}}\right) .
\end{aligned}
$$

This agrees remarkably well with the parameterization of W13, who find a slope of $5.31 \pm 0.33$, and is consistent with that of Grier et al. (2013; $5.04 \pm 0.19)$ and that of Savorgnan \& Graham $(2015 ; 6.34 \pm 0.8)$.

We find a slightly shallower slope when we use our own determinations of $\sigma_{\star}$, more consistent with that of KH13 $(4.38 \pm 0.29)$ :

$$
\begin{aligned}
\log \left(\frac{M_{\mathrm{BH}}}{M_{\odot}}\right)= & (8.66 \pm 0.09) \\
& +(4.76 \pm 0.60) \log \left(\frac{\sigma_{\star}}{200 \mathrm{~km} \mathrm{~s}^{-1}}\right)
\end{aligned}
$$

While the slopes are consistent with the literature, the intercepts are higher. Comparing Equation (2) with the parameterization by $\mathrm{W} 13$ (who find $\alpha=8.37 \pm 0.05$ ) is particularly instructive, since the slopes are almost identical. Differences between the intercepts arise from systematic differences between the sample of $\sigma_{\star}$ measurements. On average, the $\sigma_{\star}$ measurements from Atlas ${ }^{3 \mathrm{D}}$ data are lower than the literature values, causing the relation to be shifted left, thus increasing the intercept. While lower $\sigma_{\star}$ values are expected from IFS (see, e.g., KH13; B17), these measurements may be biased low if $r_{e}$ are overestimated (Section 3). Thus, the intercepts that we measure are likely too high, while the intercepts quoted in the literature are probably too low.

\subsection{The AGN Sample}

To determine the best-fitting relation for AGN, VP is used in place of $M_{\mathrm{BH}}$ :

$$
\log \left(\frac{\mathrm{VP}}{M_{\odot}}\right)=\alpha_{\mathrm{AGN}}+\beta \log \left(\frac{\sigma_{\star}}{200 \mathrm{~km} \mathrm{~s}^{-1}}\right),
$$

where, since $M_{\mathrm{BH}}=f$ VP, $\alpha_{\mathrm{AGN}}$ includes $\log f$.

The relation is parameterized with both error estimates (statistical uncertainty is used when a standard deviation is not available), and the best-fit parameters are shown in Table 3. In general, parameterizations that include the standard deviation (column (6) of Table 1) as the error in $\sigma_{\star}$ are steeper; however, they are all consistent with each other. We adopt as our best fit that which uses the statistical measurement error:

$$
\begin{aligned}
\log \left(\frac{\mathrm{VP}}{M_{\odot}}\right)= & (7.53 \pm 0.26) \\
& +(3.90 \pm 0.93) \log \left(\frac{\sigma_{\star}}{200 \mathrm{~km} \mathrm{~s}^{-1}}\right)
\end{aligned}
$$

This agrees with the parameterization for AGN found by W13 $(3.46 \pm 0.61)$, as well as for two of their quiescent galaxy subsamples: late-type galaxies $(4.23 \pm 1.26)$ and galaxies with pseudo-bulges $(3.28 \pm 1.11)$. It is also consistent with the quiescent galaxy parameterizations found in Section 4.1. The scatter in the relation is found to be $0.30 \pm 0.15 \mathrm{dex}$, which is similarly consistent with previous studies.

Table 3 also shows the best-fit parameters when the alternative definition of $\sigma_{\star}$ is used. This gives a slightly shallower slope; however, the fits are consistent between the two measures of $\sigma_{\star}$.

As with previous studies, our best fits to the AGN $M_{\mathrm{BH}^{-}} \sigma_{\star}$ relation are shallower than those for the quiescent sample. However, this does not necessarily indicate a fundamental difference between the relations for active and quiescent galaxies. $M_{\mathrm{BH}}$ is determined via different methods for the two samples, so different selection criteria apply. For quiescent galaxies it is necessary to resolve the gravitational sphere of influence of the $\mathrm{BH}$, which is not required for AGN. Recent studies have suggested that this criterion may bias the quiescent galaxy sample, and accounting for this bias substantially reduces the discrepancy between the fits (W13; Shankar et al. 2016). Expanded samples of AGN and quiescent galaxies, at both the high and low $M_{\mathrm{BH}}$ ends, are key to further investigating this difference and determining if it is physically meaningful, or simply the result of selection effects.

\subsection{The Virial Scale Factor}

We estimate $f$ by fixing the slope in Equation (4) to that for quiescent galaxies and taking the difference between the intercepts for the two samples:

$$
\log f=\alpha_{q}-\alpha_{\mathrm{AGN}}
$$

where $\alpha_{q}$ is the intercept for the quiescent galaxy sample and $\alpha_{\mathrm{AGN}}$ is the intercept for the AGN sample.

We use an adapted version of LINMIX_ERR that allows for fixing the slope and use the two quiescent galaxy parameterizations from Section 4.1, as well as that of $\mathrm{W} 13(\beta=5.31$, $\alpha=8.37$ ), to provide a comparison with the literature. W13 is chosen because the sample of $\sigma_{\star}$ that they use contains some rotation-corrected values, so it is more consistent with our sample than others available in the literature.

The results are summarized in Table 3 . The lowest value of $f$ is found with the parameterization of W13, while the highest comes from Equation (3). They are all consistent with previous estimates (e.g., Graham et al. 2011; Grier et al. 2013; Woo et al. 2015; W13), though our highest value is notably higher 
Table 3

Fits to the $M_{\mathrm{BH}}-\sigma_{\star}$ Relation

\begin{tabular}{|c|c|c|c|c|c|c|}
\hline $\begin{array}{l}\text { Sample/Fit } \\
\text { (1) }\end{array}$ & $\begin{array}{l}\sigma_{\star} \\
(2)\end{array}$ & $\begin{array}{l}\sigma_{\star} \text { Error } \\
\text { (3) }\end{array}$ & $\begin{array}{c}\alpha \\
(4)\end{array}$ & $\begin{array}{c}\beta \\
(5)\end{array}$ & $\begin{array}{c}f \\
(6)\end{array}$ & $\begin{array}{c}\epsilon \\
(7)\end{array}$ \\
\hline Quiescent & $\begin{array}{l}\text { integrated } \\
\text { spatially resolved }\end{array}$ & $\begin{array}{l}\text { measurement error } \\
\text { standard deviation }\end{array}$ & $\begin{array}{l}8.55 \pm 0.09 \\
8.66 \pm 0.09\end{array}$ & $\begin{array}{l}5.32 \pm 0.63 \\
4.76 \pm 0.60\end{array}$ & $\begin{array}{l}\cdots \\
\cdots\end{array}$ & $\begin{array}{l}0.16 \pm 0.06 \\
0.11 \pm 0.05\end{array}$ \\
\hline Active & $\begin{array}{l}\text { spatially resolved } \\
\text { spatially resolved } \\
\text { integrated }\end{array}$ & $\begin{array}{l}\text { measurement error } \\
\text { standard deviation } \\
\text { measurement error }\end{array}$ & $\begin{array}{l}7.53 \pm 0.26 \\
7.55 \pm 0.26 \\
7.38 \pm 0.25\end{array}$ & $\begin{array}{l}3.90 \pm 0.93 \\
4.00 \pm 0.94 \\
3.53 \pm 0.93\end{array}$ & $\begin{array}{l}\cdots \\
\cdots \\
\cdots\end{array}$ & $\begin{array}{l}0.30 \pm 0.15 \\
0.27 \pm 0.16 \\
0.34 \pm 0.18\end{array}$ \\
\hline Equation (2) & spatially resolved & standard deviation & $7.86 \pm 0.15$ & 5.32 & $4.94 \pm 1.75$ & $0.27 \pm 0.16$ \\
\hline Equation (2) & integrated & measurement error & $7.77 \pm 0.17$ & 5.32 & $6.05 \pm 2.45$ & $0.43 \pm 0.21$ \\
\hline Equation (3) & spatially resolved & measurement error & $7.73 \pm 0.14$ & 4.76 & $8.49 \pm 2.77$ & $0.29 \pm 0.16$ \\
\hline Equation (3) & spatially resolved & standard deviation & $7.72 \pm 0.14$ & 4.76 & $8.67 \pm 2.89$ & $0.24 \pm 0.14$ \\
\hline Equation (3) & integrated & measurement error & $7.64 \pm 0.16$ & 4.76 & $10.37 \pm 3.86$ & $0.37 \pm 0.18$ \\
\hline W13 & spatially resolved & measurement error & $7.86 \pm 0.15$ & 5.31 & $3.23 \pm 1.14$ & $0.33 \pm 0.16$ \\
\hline
\end{tabular}

Notes. Column 1: the first five rows give the sample being fitted, either quiescent or active, the rest show the parameterization being used. Column 2: method by which $\sigma_{\star}$ was determined, either from an average of spatially resolved spectra, or from a single integrated spectrum. Column 3: uncertainty in $\sigma_{\star}$ used in the fit. Column 4: the intercepts. Column 5: the slopes. Column 6: calculated $f$ value. Column 7: scatter in the relation.

than most quoted in the literature. Our determinations are also consistent with the results of dynamical modeling of the BLR by Pancoast et al. (2014), who modeled five active galaxies (including NGC 5548 and NGC 6814) and determined $f$ separately for each, finding a mean of $\langle\log f\rangle=0.68 \pm 0.40$.

For comparison we perform the same fitting using the alternative definition of $\sigma_{\star}$, which are listed in Table 3. They are generally steeper, but consistent within the errors.

As can be seen, $f$ varies significantly depending on the chosen parameterization of the quiescent $M_{\mathrm{BH}}-\sigma_{\star}$ relation, and this is driven by the different measurements of $\sigma_{\star}$ for the quiescent sample. Given the previously discussed issues with the $r_{e}$ determinations for the quiescent sample, it is clear that the best value to use for $f$ is still not settled.

IFS provides more information about the galaxy kinematics, so IFS-based $\sigma_{\star}$ measurements are an improvement over previous estimates. The quiescent galaxy parameterizations presented in this work rely on such estimates and provide a more consistent basis for comparison with our AGN sample, so $f$ values determined from these parameterizations are preferable. Equation (3) should provide the best parameterization to use with the AGN sample, since the $\sigma_{\star}$ measurements do not include contributions from the rotational velocity. However, the bias that arises from poorly constrained $r_{e}$ measurements for the quiescent sample does not affect the AGN sample, so they are not completely consistent. Following the discussion in Section 4.1, it is reasonable to consider that the $f$ value obtained from Equation (3) is too high, and the $f$ value obtained from the parameterization of $\mathrm{W} 13$ is too low, so we recommend $f=4.82 \pm 1.67$. This is close to the median $f$ value of all those listed in Table 3, though the scatter among those values is 2.6, which is higher than the quoted uncertainty.

Figure 2 shows the $M_{\mathrm{BH}}-\sigma_{\star}$ relation, with the lines of best fit, for both samples. While the slopes are different, the samples clearly overlap, and the absence of AGN with high $M_{\mathrm{BH}}$ and high $\sigma_{\star}$ may well be responsible for the difference in slopes. The AGN sample is split into barred and unbarred galaxies (red and black points, respectively), since previous studies have suggested morphological dependencies in the $M_{\mathrm{BH}^{-}} \sigma_{\star}$ relation.

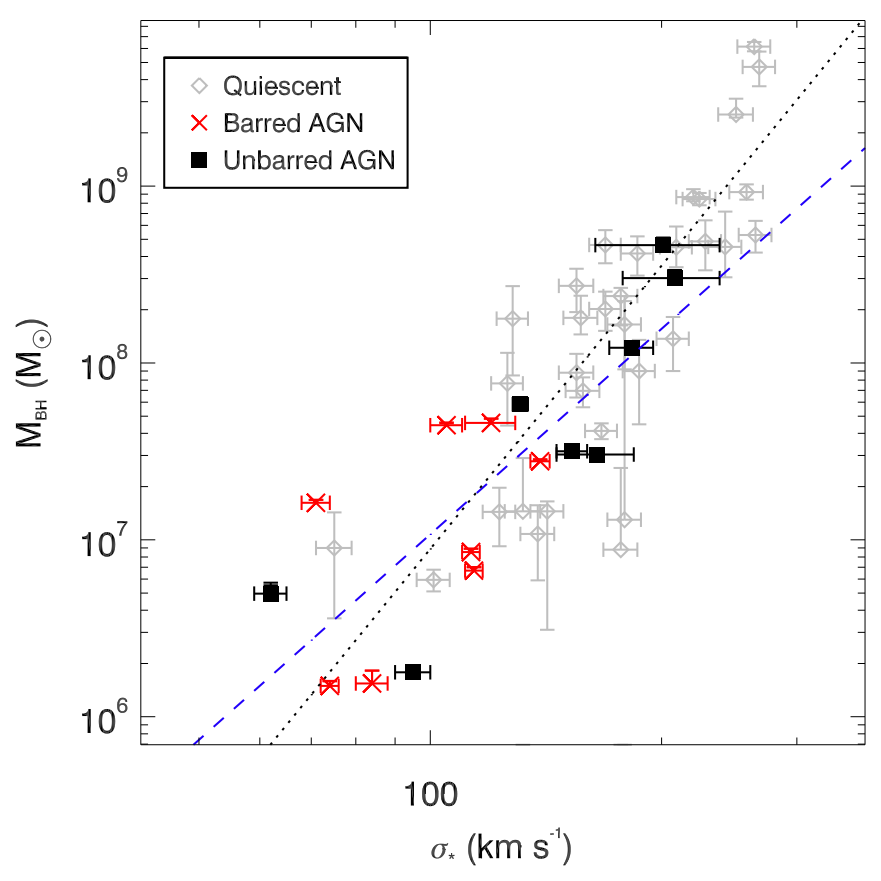

Figure 2. $M_{\mathrm{BH}^{-}} \sigma_{\star}$ relation for quiescent galaxies (gray) and AGN (red for unbarred and black for barred). The adopted best fit for the quiescent sample is shown as the dotted line and for the AGN sample is the dashed line. VPs are converted to $M_{\mathrm{BH}}$ using $f=4.82$.

We see no obvious difference between these subsamples, though we caution that this sample is too small to draw any definite conclusions.

\section{Summary}

We have presented a recalibration of the $M_{\mathrm{BH}}-\sigma_{\star}$ relation, using $\sigma_{\star}$ determinations from IFS. Our results can be summarized as follows.

(i) Both the quiescent and AGN samples are fitted using two different definitions of $\sigma_{\star}$, and we find that including 
rotational broadening tends to produce a flatter slope. Our slopes are consistent with previous studies, as is the fact that fits to the AGN sample are consistently shallower than the quiescent galaxy parameterizations.

(ii) The intercepts in our quiescent fits are larger than those in the literature, due to systematically lower $\sigma_{\star}$ estimates. While this is expected for measurements from IFS, the quiescent sample suffers from poorly constrained $r_{e}$ determinations that may bias $\sigma_{\star}$ estimates low. This problem impacts the majority of studies in the literature. Larger intercepts result in larger $f$ values, demonstrating the sensitivity of $f$ to the details of the $\sigma_{\star}$ measurements. We recommend $f=4.82 \pm 1.67$, but caution that there remain potentially significant biases that must be addressed.

(iii) Along with more accurate determinations of $r_{e}$, this work demonstrates the need for a significantly expanded sample of active and quiescent galaxies with $\sigma_{\star}$ from IFS and accurately constrained $r_{e}$. This analysis clearly demonstrates that we are now in a regime where the details of the $\sigma_{\star}$ determination are important.

M.C.B. gratefully acknowledges support from the NSF through CAREER grant AST-1253702 to Georgia State University. S.R. and M.V. gratefully acknowledge financial support from the Danish Council for Independent Research via grant No. DFF 4002-00275. We would like to thank the anonymous referee, whose comments have served to significantly improve the Letter.

\section{References}

Batiste, M., Bentz, M. C., Manne-Nicholas, E. R., Onken, C. A., \& Bershady, M. A. 2017, ApJ, 835, 271

Beifiori, A., Courteau, S., Corsini, E. M., \& Zhu, Y. 2012, MNRAS, 419, 2497

Bellovary, J. M., Holley-Bockelmann, K., Gültekin, K., et al. 2014, MNRAS, 445,2667
Bentz, M. C., Cackett, E. M., Crenshaw, D. M., et al. 2016, ApJ, 830, 136

Bentz, M. C., Denney, K. D., Grier, C. J., et al. 2013, ApJ, 767, 149

Bentz, M. C., Horenstein, D., Bazhaw, C., et al. 2014, ApJ, 796, 8

Bentz, M. C., \& Katz, S. 2015, PASP, 127, 67

Bentz, M. C., Peterson, B. M., Netzer, H., Pogge, R. W., \& Vestergaard, M. 2009, ApJ, 697, 160

Blandford, R. D., \& McKee, C. F. 1982, ApJ, 255, 419

Brown, J. S., Valluri, M., Shen, J., \& Debattista, V. P. 2013, ApJ, 778, 151

Cappellari, M., \& Emsellem, E. 2004, PASP, 116, 138

Cappellari, M., Emsellem, E., Krajnović, D., et al. 2011, MNRAS, 413, 813

Cappellari, M., Scott, N., Alatalo, K., et al. 2013, MNRAS, 432, 1709

Falcón-Barroso, J., Lyubenova, M., van de Ven, G., et al. 2017, A\&A, 597, 48

Ferrarese, L., \& Merritt, D. 2000, ApJL, 539, L9

Gebhardt, K., Bender, R., Bower, G., et al. 2000, ApJL, 539, L13

Graham, A. W. 2008, ApJ, 680, 143

Graham, A. W. 2016, in Galactic Bulges, Vol. 418 (Cham: Springer International), 263

Graham, A. W., Onken, C. A., Athanassoula, E., \& Combes, F. 2011, MNRAS, 412, 2211

Grier, C. J., Martini, P., Watson, L. C., et al. 2013, ApJ, 773, 90

Gültekin, K., Richstone, D. O., Gebhardt, K., et al. 2009, ApJ, 698, 198

Hartmann, M., Debattista, V. P., Cole, D. R., et al. 2014, MNRAS, 441, 1243

Hu, J. 2008, MNRAS, 386, 2242

Kang, W.-R., Woo, J.-H., Schulze, A., et al. 2013, ApJ, 767, 26

Kelly, B. C. 2007, ApJ, 665, 1489

Kormendy, J., Fisher, D. B., Cornell, M. E., \& Bender, R. 2009, ApJS, 182,216

Kormendy, J., \& Ho, L. C. 2013, ARA\&A, 51, 511

McConnell, N. J., \& Ma, C.-P. 2013, ApJ, 764, 184

Onken, C. A., Ferrarese, L., Merritt, D., et al. 2004, ApJ, 615, 645

Pancoast, A., Brewer, B. J., Treu, T., et al. 2014, MNRAS, 445, 3073

Park, D., Kelly, B. C., Woo, J.-H., \& Treu, T. 2012, ApJS, 203, 6

Peng, C. Y., Ho, L. C., Impey, C. D., \& Rix, H.-W. 2002, AJ, 124, 266

Peng, C. Y., Ho, L. C., Impey, C. D., \& Rix, H.-W. 2010, AJ, 139, 2097

Raimundo, S. I., Davies, R. I., Gandhi, P., et al. 2013, MNRAS, 431, 2294

Savorgnan, G. A. D., \& Graham, A. W. 2015, MNRAS, 446, 2330

Shankar, F., Bernardi, M., Sheth, R. K., et al. 2016, MNRAS, 460, 3119

van den Bosch, R. 2016, ApJ, 831, 134

Williams, M. J., Bureau, M., \& Cappellari, M. 2010, MNRAS, 409, 1330

Woo, J.-H., Schulze, A., Park, D., et al. 2013, ApJ, 772, 49

Woo, J.-H., Treu, T., Barth, A. J., et al. 2010, ApJ, 716, 269

Woo, J.-H., Yoon, Y., Park, S., Park, D., \& Kim, S. C. 2015, ApJ, 801, 38 